\title{
The Effect of Early Life Antibiotic Exposures on Diarrheal Rates Among Young Children in Vellore, India
}

\author{
Elizabeth T. Rogawski, PhD, * Daniel Westreich, PhD, * Sylvia Becker-Dreps, MD, † Linda S. Adair, PhD, $\neq$ \\ Robert S. Sandler, MD, *§ Rajiv Sarkar, PhD, $ๆ$ Deepthi Kattula, BDS, MSc, 9 Honorine D. Ward, MBBS,\| \\ Steven R. Meshnick, MD, PhD, * and Gagandeep Kang, MD, PhD
}

\begin{abstract}
Background: Antibiotic treatment of childhood illnesses is common in India. In addition to contributing to antimicrobial resistance, antibiotics might result in increased susceptibility to diarrhea through interactions with the gastrointestinal microbiota. Breast milk, which enriches the microbiota early in life, may increase the resilience of the microbiota against perturbations by antibiotics. Methods: In a prospective observational cohort study, we assessed whether antibiotic exposures from birth to 6 months affected rates of diarrhea up to age 3 years among 465 children from Vellore, India. Adjusting for treatment indicators, we modeled diarrheal rates among children exposed and unexposed to antibiotics using negative binomial regression. We further assessed whether the effect of antibiotics on diarrheal rates was modified by exclusive breastfeeding at 6 months.

Results: More than half of the children $(n=267,57.4 \%)$ were given at least one course of antibiotics in the first 6 months of life. The adjusted relative incidence rate of diarrhea was $33 \%$ higher among children who received antibiotics under 6 months of age compared with those who did not (incidence rate ratio: $1.33,95 \%$ confidence interval: $1.12,1.57$ ). Children who were exclusively breastfed until 6 months of age did not have increased diarrheal rates following antibiotic use.
\end{abstract}

Conclusions: Antibiotic exposures early in life were associated with increased rates of diarrhea in early childhood. Exclusive breastfeeding might protect against this negative impact.

Key Words: antimicrobials, diarrhea, microbiota, India

(Pediatr Infect Dis J 2015;34:583-588)

$\mathrm{A}^{\mathrm{s}}$ ntibiotics are frequently used to treat childhood illnesses, especially respiratory tract infections, otitis media and diarrhea. However, many of these infections are viral and/or self-limiting, such that antibiotics are not necessary. ${ }^{1-3}$ The overuse of antibiotics among children has been reported around the world, ${ }^{1,4,5}$ and antibiotic over-prescribing by primary care physicians ${ }^{6,7}$ in India is

Accepted for publication November 17, 2014.

From the *Department of Epidemiology, †Department of Family Medicine, $\$$ Department of Nutrition, §Department of Medicine, UNC-Chapel Hill, North Carolina; qDivision of Gastrointestinal Sciences, Christian Medical College, Vellore, India; and IDivision of Geographic Medicine and Infectious Diseases, Tufts Medical Center, Boston, Massachusetts.

This study was supported by the National Institute of Allergy and Infectious Diseases at the National Institutes of Health [5-R01-AI072222; 5-T32-AI070114-08; D43-TW007392). S.B.D. has had investigator-initiated research grants with Pfizer, Inc. and Merck, Sharp and Dohme for studies unrelated to the submitted work. The other authors have no conflicts of interest or funding to disclose.

Address for correspondence: Elizabeth T. Rogawski, PhD, Department of Epidemiology, 3113 Michael Hooker Research Center, Campus Box 7435, UNCChapel Hill, North Carolina 27599-7435, E-mail: rogawski@unc.edu

Copyright (C) 2015 Wolters Kluwer Health, Inc. All rights reserved. This is an open access article distributed under the terms of the Creative Commons Attribution-NonCommercial-NoDerivatives 3.0 License, where it is permissible to download and share the work provided it is properly cited. The work cannot be changed in any way or used commercially.

ISSN: 0891-3668/15/3406-0583

DOI: $10.1097 /$ INF.0000000000000679 further compounded by the ability to purchase antibiotics over the counter without a prescription ${ }^{8}$ in spite of government regulations. ${ }^{9}$

Often, the primary rationale given for restricting antibiotic use is to slow the development of antimicrobial resistance. ${ }^{10,11}$ However, recent evidence has suggested antibiotics may also have a direct negative impact on the patients prescribed the drugs, primarily through interactions with the gastrointestinal (GI) microbiota. ${ }^{11}$ The development of the GI microbiota in the first few months of life coincides with a critical period of intestinal structure and immune system maturation. ${ }^{12-14} \mathrm{~A}$ healthy microbiota is important in the early life defense against GI infections by providing a barrier effect that inhibits the attachment and growth of pathogens. ${ }^{15,16}$ Antibiotics impact the diversity and composition of the GI microbiota, and some of these effects can persist long after treatment is completed, ${ }^{17,18}$ especially among infants. ${ }^{19-23}$ These exposures have been further associated with impaired GI functioning, intestinal inflammation, and increased intestinal permeability and risk of systemic infections. ${ }^{21,24,25}$

Given the potential negative impact of antibiotics on the developing microbiota, we assessed whether antibiotic exposure in the first 6 months of life affected subsequent rates of all-cause diarrhea from 6 months to 3 years of age in a birth cohort from Vellore, India. We also explored the impact of exclusive breastfeeding, which may modify this effect given the beneficial role of breast milk on the microbiota. ${ }^{26-28}$

\section{MATERIALS AND METHODS}

We performed an analysis of data from a prospective observational cohort study of immune responses in cryptosporidiosis in 497 children followed from birth to 3 years of age. The parent study population consisted of all children born in 4 geographically adjacent, semiurban slums of Vellore, in the state of Tamil Nadu, India, between April 2009 and May 2010. The study population, enrollment strategy and data collection methods have been previously described. ${ }^{29}$ The study was approved by the Institutional Review Boards of the Christian Medical College, Vellore, India, Tufts University Health Sciences campus, Boston, USA and University of North Carolina-Chapel Hill, USA.

Briefly, baseline information on maternal demographic characteristics, socioeconomic indicators, health-seeking behavior, environment, diet and characteristics of delivery were collected within 45 days of birth. Fieldworkers visited households of all enrolled children twice per week until 3 years of age to collect information on incidence, duration and hospitalization for all illnesses as well as specific severity and treatment details for reported diarrhea. Caregivers were asked specifically if any antibiotics were given for diarrhea (yes/no) and if so, to report the name of the antibiotic (available for $64.0 \%$ of antibiotic reports). Breastfeeding history (exclusive, nonexclusive, none) was collected every 2 weeks until breastfeeding was stopped completely. Height and weight were measured monthly at the study clinic. Antibiotic prescriptions and corresponding diagnoses were also extracted from records at the physician-run study clinic that was established in the study area to provide free health care to study children. 


\section{Data and Definitions}

The main exposures were any antibiotic exposure in the first 6 months of life and the total number of antibiotic courses in the first 6 months of life, both based on a combination of antibiotic prescriptions recorded in clinic records and caregiver-reported antibiotic treatment at birth and for diarrhea. We excluded all topical antibiotics (neosporin, neomycin, soframycin and gentian violet). This clinical definition of antibiotic exposure was used in all primary analyses. Exclusive breastfeeding was defined according to the standard World Health Organization (WHO) definition ${ }^{30}$ as feeding with breast milk only with the exception of vitamins, mineral supplements and medicines (no liquid, semisolid or solid food).

Diarrhea outcomes were based on caregiver-reported diarrhea at twice-weekly home visits. Diarrhea was defined using the standard WHO definition as at least 3 loose or watery stools in a 24-hour period. ${ }^{31} \mathrm{~A}$ new episode was defined as diarrhea that occurred after at least 2 days of normal bowel movements. Rates of diarrhea after 6 months of age per child were defined by the total number of incident episodes divided by the total time that child remained in the study. We excluded from person-time denominators days with diarrhea (when a child was not at risk of incident diarrhea, $0.3 \%$ of total person-time from 6 months to 3 years), periods during which the child was unreachable $(0.6 \%$ of person-time from 6 months to 3 years $)$ and any time after loss to follow-up or death ( $8.2 \%$ of person-time). Because the proportion of missing data was less than $3 \%$ for all baseline variables (see footnote in Table 1), we imputed the median values of variables for individuals with missing data.

\section{Data Analysis}

We used Poisson and negative binomial regression to model the rates of diarrhea from 6 months to 3 years of age. The negative binomial model was preferred over the Poisson model by likelihood ratio test $(P<0.0001)$ and was used in final analyses to correct for overdispersion. ${ }^{34}$ Crude and adjusted incidence rate ratios (IRRs) for diarrhea were estimated comparing children who were exposed to early life antibiotics with those who were not. Confounding variables were chosen by causal directed acyclic graph ${ }^{35}$ based on the substantive literature, and optimal variable coding was determined by likelihood ratio test $(\alpha=0.1)$ and Akaike's information criterion.

Using the causal directed acyclic graph, we identified the following demographic characteristics and measures of illness in the first 6 months for adjustment in the models: child sex, socioeconomic status based on the Kuppuswamy scale, ${ }^{32}$ maternal education, household hygiene, ${ }^{33}$ household crowding, low birth weight $(<2.5 \mathrm{~kg})$, number of diarrhea episodes in first 6 months, total number of days with diarrhea in first 6 months, maximum Vesikari score ${ }^{36}$ of any diarrhea episode in first 6 months, number of severe episodes in first 6 months (Vesikari $\geq 11$ ), prolonged or persistent diarrhea episode in first 6 months, hospitalization for diarrhea in the first 6 months, fever during diarrhea in first 6 months, dehydration during diarrhea in first 6 months, underweight [average weight-for-age $\mathrm{Z}$ score under 6 months of age $<-2$ standard deviations (SD) from the $2006 \mathrm{WHO}$ growth reference ${ }^{37}$ ], stunting (average height-for-age $\mathrm{Z}$ score $<-2 \mathrm{SD}$ ), wasting (average weightfor-height $Z$ score $<-2$ SD) in the first 6 months, any severe illness in first 6 months and number of other infections in first 6 months.

\section{Effect Measure Modification}

We assessed whether the effect of early life antibiotic exposure on diarrheal risk after 6 months was modified by exclusive breastfeeding at 6 months of age or at first antibiotic exposure by reporting stratum-specific estimates and testing homogeneity by likelihood ratio test $(\alpha=0.1)$. We further explored the role of breastfeeding by assessing the crude association between exclusive breastfeeding and antibiotic treatment (both any exposure under 6 months, using logrisk regression, and age at first exposure, using log-transformed age with linear regression). We further assessed effect measure modification by sex, Cesarean section birth, age at first diarrhea and growth status (underweight, stunted and wasted) in first 6 months.

\section{Sensitivity Analyses}

To assess potential misclassification of the exposure, we repeated main analyses with more restricted definitions of antibiotic exposure that included caregiver-reported antibiotics only if an antibiotic name was recorded. To determine if the effect of antibiotics on diarrheal rates differed by antibiotic type, ${ }^{38,39}$ we repeated analyses separately comparing children who exclusively received one of the most commonly used antibiotics, amoxicillin and cotrimoxazole, with children who received no antibiotics. We were unable to assess other major classes of antibiotics because few children received other drugs. We further assessed if the effect of antibiotics differed depending on (1) the indication for which antibiotic treatment was given; (2) the number of diarrhea episodes experienced in the first 6 months of life; and (3) the time period for diarrheal outcomes (6-18 months of age compared with 18-36 months).

\section{RESULTS}

We included 465 of 497 children in the parent cohort (93.6\%) who remained in the study for more than 6 months and were therefore at risk for diarrhea after 6 months of age. An additional 11.8\% of children were lost to follow-up over the remaining study period; 21, 24 and 10 children dropped out before 1,2 and 3 years of age, respectively. All person-time during which these children remained in the study was included in the analysis, and differential follow-up times were accounted for in the analytic models. Six children died after 6 months of age, and 2 of these deaths were associated with diarrhea. Two-thirds of children were of low socioeconomic status ( $n=308,66.2 \%$, Table 1). Children living in crowded households with more than 4 people per room were common $(n=149,32.0 \%)$, and approximately half of the mothers reported poor household hygiene $(n=221,47.5 \%)$. By 6 months of age, most children had stopped exclusive breastfeeding $(n=394,84.7 \%)$ and had their first episode of diarrhea $(n=300,64.5 \%)$.

Rates of diarrhea were highest between 3 and 9 months of life, with crude incidence rates of 28.1 cases per 100 person-months among children aged 3-6 months and 30.3 cases per 100 personmonths among children aged 6-9 months. After 6 months of age, diarrhea rates decreased to an average rate of 13.4 cases per 100 person-months from 6 months to 3 years, corresponding to a total of 1693 episodes, or an average of 3.6 episodes per child in that period. The median number of diarrhea episodes after 6 months of age was 2 (interquartile range: 1, 5; Fig. 1).

Antibiotic exposure in the first 6 months of life was common. More than half $(n=267,57.4 \%)$ of children were given at least one course of antibiotics in the first 6 months (Table 1). At the study clinic, antibiotic prescriptions in this age group were most frequently associated with diagnoses for upper respiratory infections ( $\mathrm{n}=160,36.5 \%$ of total prescriptions), acute gastroenteritis (including diarrhea; $\mathrm{n}=111,25.3 \%$ ), lower respiratory infections $(\mathrm{n}=71,16.2 \%)$ and acute otitis media $(\mathrm{n}=18,4.1 \%)$. Almost all children $(n=415,89.2 \%)$ also received antibiotics after 6 months of age, and children who were given antibiotics under 6 months were more likely to receive antibiotics after 6 months compared with children who were not given antibiotics under 6 months (Table 1).

Amoxicillin and sulfamethoxazole/trimethoprim (cotrimoxazole) were the most commonly given antibiotics. Children were generally given amoxicillin for respiratory illnesses and cotrimoxazole for GI illnesses. Approximately one-third of children received either 
TABLE 1. Demographic Characteristics of 465 Children in a Birth Cohort in Vellore, Tamil Nadu, India 2009-2013

\begin{tabular}{|c|c|c|}
\hline & $\begin{array}{l}\text { No Antibiotics 0-5 Months } \\
(\mathrm{n}=198)\end{array}$ & $\begin{array}{l}\text { Antibiotics 0-5 Months } \\
\qquad(\mathrm{n}=267)\end{array}$ \\
\hline & No. of Children (\%) & No. of Children (\%) \\
\hline \multicolumn{3}{|l|}{ Household characteristics } \\
\hline \multicolumn{3}{|l|}{ Socioeconomic status* } \\
\hline Low & $139(70.2)$ & $169(63.3)$ \\
\hline Medium & $56(28.3)$ & $93(34.8)$ \\
\hline High & $3(1.5)$ & $5(1.9)$ \\
\hline \multicolumn{3}{|l|}{ Maternal education } \\
\hline No formal education & $71(35.9)$ & $99(37.1)$ \\
\hline Primary/middle school & $72(36.4)$ & $86(32.2)$ \\
\hline Higher secondary school & $50(25.3)$ & $73(27.3)$ \\
\hline $\begin{array}{l}\text { College/polytechnic/professional } \\
\text { school }\end{array}$ & $5(2.5)$ & $9(3.4)$ \\
\hline Poor household hygiene $\dagger$ & $89(45.0)$ & $135(50.6)$ \\
\hline \multicolumn{3}{|l|}{ Crowding } \\
\hline Low $(\leq 3$ people/room $)$ & 39 (19.7) & $96(36.0)$ \\
\hline Medium (3.1-4 people/room) & $90(45.5)$ & $91(34.1)$ \\
\hline High ( $>4$ people/room $)$ & $69(34.9)$ & $80(30.0)$ \\
\hline \multicolumn{3}{|l|}{ Child characteristics } \\
\hline \multicolumn{3}{|l|}{ Sex of child } \\
\hline Male & $97(49.0)$ & $149(55.8)$ \\
\hline Female & $101(51.0)$ & $118(44.2)$ \\
\hline Cesarean section & $32(16.2)$ & $50(18.7)$ \\
\hline Low birth weight $\neq$ & $36(18.2)$ & $42(15.7)$ \\
\hline Baby kept in ICU at birth & $10(5.1)$ & $23(8.6)$ \\
\hline Antibiotics at birth $\ddagger$ & 0 & $12(4.5)$ \\
\hline \multicolumn{3}{|l|}{ Age at first diarrhea } \\
\hline$<6$ months & $100(50.5)$ & $200(74.9)$ \\
\hline 6 months to 1 year & $58(29.3)$ & $38(14.2)$ \\
\hline$>1$ year & $17(8.6)$ & $14(5.2)$ \\
\hline No diarrhea & $23(11.6)$ & $15(5.6)$ \\
\hline \multicolumn{3}{|l|}{ Number of diarrhea episodes $0-5$ months } \\
\hline 0 & $98(49.5)$ & $67(25.1)$ \\
\hline 1 & $52(26.3)$ & $75(28.1)$ \\
\hline 2 & $34(17.2)$ & $68(25.5)$ \\
\hline $3+$ & $14(7.1)$ & $57(21.4)$ \\
\hline $\begin{array}{l}\text { Age (months) at stopping exclusive } \\
\text { breastfeeding (mean, SD) }\end{array}$ & $4.0(2.20)$ & $3.9(1.98)$ \\
\hline $\begin{array}{l}\text { Age (months) at stopping all breast- } \\
\text { feeding (mean, SD) }\end{array}$ & $16.8(7.95)$ & $16.7(8.57)$ \\
\hline Underweight in first 6 months & $50(25.3)$ & $74(27.7)$ \\
\hline Stunted in first 6 months & $34(17.2)$ & $67(25.1)$ \\
\hline Wasted in first 6 months & $34(17.2)$ & $38(14.2)$ \\
\hline \multicolumn{3}{|l|}{ Antibiotic use in first 6 months } \\
\hline 1 & - & $135(50.6)$ \\
\hline 2 & - & $74(27.7)$ \\
\hline $3+$ & _- & $58(21.7)$ \\
\hline \multicolumn{3}{|l|}{ Antibiotic use 6 months to 3 years } \\
\hline 1 & $23(11.6)$ & $16(6.0)$ \\
\hline 2 & $27(13.6)$ & $22(8.2)$ \\
\hline $3+$ & $118(59.6)$ & $209(78.3)$ \\
\hline
\end{tabular}

or both of these antibiotics before 6 months of age. Less common antibiotic exposures were cefixime $(4.3 \%$ of children received cefixime under 6 months of age), fluoroquinolones (ciprofloxacin, norfloxacin and levofloxacin; $2.4 \%$ ) and azithromycin (1.7\%).

\section{Effect of Antibiotics on Diarrheal Rates}

Antibiotic exposure under 6 months of age was crudely associated with an increase in diarrhea rates from 6 months to 3 years (Table 2). After multivariable adjustment, the relative incidence rate of diarrhea was $33 \%$ higher among children who had at least 1 course of antibiotics under 6 months of age compared with those who did not [IRR: $1.33,95 \%$ confidence interval (CI): 1.12, 1.57]. There was no significant difference in the effect by the number of antibiotic courses received. Children who received 3 or more courses of antibiotics had a slightly lower relative increase compared with 1 or 2 courses, though this estimate was less precise (Table 2).

\section{Effect Measure Modification}

The effect of early antibiotic exposure differed by exclusive breastfeeding practices in the first 6 months of life ( $P$ for 
FIGURE 1. Number of diarrhea episodes after 6 months of age among 465 children in a birth cohort in Vellore, Tamil Nadu, India 2009-2013.

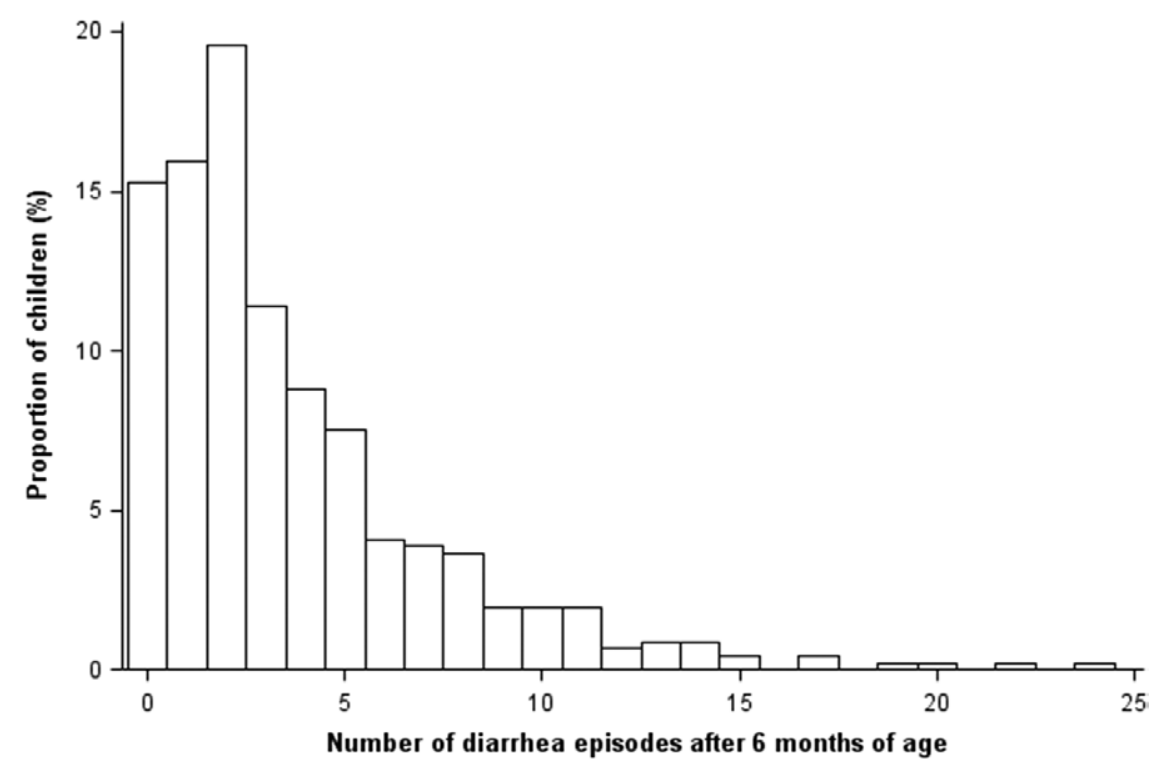

\section{Sensitivity Analysis}

\section{Exposure Misclassification}

In an alternative definition of antibiotic exposure, we included caregiver-report only if an antibiotic was named. Adjusted effects were qualitatively similar, but slightly closer to the null, for both overall effects and effects stratified by exclusive breastfeeding.

\section{Antibiotic Type}

There were no differences in the effect on diarrheal rates by antibiotic drug type. A sufficient number of children received amoxicillin $(n=145)$ and cotrimoxazole $(n=158)$ for multivariate analyses. The adjusted IRR comparing children exposed to only amoxicillin with children who received no antibiotics was 1.46 (95\% CI: 1.12, 1.90), which was similar to the rate ratio comparing children exposed to only cotrimoxazole with unexposed children: 1.32 (95\% CI: $1.04,1.69)$.

\section{Indication for Treatment, Number of Diarrhea Episodes and Time Period for Diarrheal Outcomes}

There was no difference in the effect of antibiotics on diarrheal rates depending on the indicating illness for antibiotic treatment. Children treated with antibiotics for only diarrheal and only non-diarrheal illnesses had similar increases in diarrhea rates after 6 months of age. The effect was also the same regardless of the number of diarrhea episodes experienced during the first 6 months and

TABLE 2. Estimated Effect of Antibiotic Exposure in the First 6 Months of Life on Incident Rates of Diarrhea From 6 Months to 3 Years of Age Among 465 Children in a Birth Cohort in Vellore, Tamil Nadu, India 2009-2013

\begin{tabular}{|c|c|c|c|c|c|}
\hline & & \multirow[b]{2}{*}{ No. of Children } & \multirow[b]{2}{*}{ Rate of Diarrhea* } & \multicolumn{2}{|c|}{ Incidence Rate Ratio (95\% CI) } \\
\hline & & & & Crude & Adjusted $\dagger$ \\
\hline \multirow[t]{2}{*}{ Antibiotics $<6$ months } & No & 198 & 10.1 & 1. & 1. \\
\hline & Yes & 267 & 15.9 & $1.58(1.33,1.88)$ & $1.33(1.12,1.57)$ \\
\hline \multirow[t]{4}{*}{ Number of antibiotic courses } & 0 & 198 & 10.1 & 1 & 1. \\
\hline & 1 & 135 & 15.0 & $1.49(1.22,1.83)$ & $1.36(1.12,1.64)$ \\
\hline & 2 & 74 & 16.7 & $1.66(1.30,2.11)$ & $1.35(1.07,1.70)$ \\
\hline & $3+$ & 58 & 17.0 & $1.69(1.28,2.22)$ & $1.17(0.89,1.55)$ \\
\hline
\end{tabular}

*Rate per 100 person-months.

†Incident rate ratio adjusted for covariates listed in the methods. 
TABLE 3. Estimated Effect of Antibiotic Exposure in the First 6 Months of Life on Rates of Diarrhea From 6 Months to 3 Years of Age by Exclusive Breastfeeding at 6 Months of Age Among 465 Children in a Birth Cohort in Vellore, Tamil Nadu, India 2009-2013

\begin{tabular}{|c|c|c|c|}
\hline & & $\begin{array}{l}\text { No. of } \\
\text { Children }\end{array}$ & $\begin{array}{l}\text { Adjusted IRR* } \\
\quad(95 \% \mathrm{CI})\end{array}$ \\
\hline \multicolumn{4}{|c|}{ Exclusively breastfed at 6 months $(\mathrm{n}=71)$} \\
\hline \multirow[t]{2}{*}{ Antibiotics $<6$ months } & No & 28 & 1. \\
\hline & Yes & 43 & $0.76(0.50,1.13)$ \\
\hline \multirow[t]{3}{*}{ Number of antibiotic courses $\dagger$} & 0 & 28 & 1. \\
\hline & 1 & 20 & $0.73(0.45,1.19)$ \\
\hline & $2+$ & 23 & $0.77(0.48,1.23)$ \\
\hline \multicolumn{4}{|c|}{ Not exclusively breastfed at 6 months $(\mathrm{n}=394)$} \\
\hline \multirow[t]{2}{*}{ Antibiotics $<6$ months } & No & 170 & 1. \\
\hline & Yes & 224 & $1.48(1.23,1.78)$ \\
\hline \multirow[t]{3}{*}{ Number of antibiotic courses $\dagger$} & 0 & 170 & 1 \\
\hline & 1 & 115 & $1.53(1.24,1.88)$ \\
\hline & $2+$ & 109 & $1.42(1.14,1.77)$ \\
\hline
\end{tabular}

* Incident rate ratio adjusted for covariates listed in the methods.

$\dagger$ Categorization was reduced to 0,1 and $2+$ courses because of small sample size (reduced model is supported by Akaike's information criterion)

the time period for diarrheal outcomes (6-18 months of age compared with 18-36 months). Results for all sensitivity analyses are available to readers by email request to the corresponding author.

\section{DISCUSSION}

Our study provides the first evidence that antibiotic treatment early in life is associated with increased rates of all-cause diarrhea from 6 months to 3 years of age, even when controlling for indications for treatment, such as illness burden and severity. Furthermore, we found that exclusive breastfeeding during antibiotic exposure may be protective against the effects of antibiotics because antibiotic exposure was not associated with increased diarrheal rates among children who were exclusively breastfed for at least the first 6 months of life.

The effect of antibiotic treatment on diarrheal risk may be mediated by a prolonged effect of the antibiotics on microbiota composition $^{17}$ or through collateral effects on intestinal structure and function relating to inflammation, permeability and intestinal immunity. ${ }^{24,40}$ In this way, antibiotics could affect susceptibility to diarrhea due to diverse pathogen etiologies. The strong effect modification by exclusive breastfeeding may also be explained by interactions with the microbiota. Bacteria, such as Lactobacillus, are present in breast milk and are thought to be beneficial: in a randomized trial of a Lactobacillus strain present in breast milk, infants given the probiotic demonstrated reduced incidence of GI infections. ${ }^{28,41}$ The protective effect of breastfeeding suggests the microbiota enriched by breast milk may be more resistant to perturbations by antibiotics.

Antibiotic treatment in the first 6 months of life was common among study children $(57.4 \%)$. In the US and UK, the proportion of children treated under 6 months of age based on caregiver-report is reported to be lower, at approximately one third of children. ${ }^{42,43}$ Higher rates of antibiotic use in our study population may be because of higher rates of infection, better capture of antibiotic prescriptions in clinic records and greater availability of antibiotics without prescriptions.

The main limitation of this study was potential under-ascertainment of antibiotic exposures for antibiotics obtained outside of the study clinic for non-diarrheal illnesses. However, we expect almost all antibiotic exposures to be captured in clinic records because the study clinic was conveniently located in the residential area where study children lived and provided clinical care and medicines free of charge. High concordance between caregiver-reported and antibiotic prescriptions for diarrhea supports our assumption that most antibiotic exposures were recorded in clinic records (78\% of antibiotic prescriptions during diarrhea episodes were associated with caregiverreported antibiotic treatment). The validity of caregiver-reported antibiotics was supported in sensitivity analyses by consistent results when using a more restricted definition of antibiotic exposure that required caregivers to list an antibiotic name.

We also did not have information on the duration of antibiotic exposures. Length of therapy may be an important component of the effect of antibiotics on diarrheal risk given the potential for longer antibiotic exposure periods to have increased impact on the microbiota. ${ }^{17,18,38}$ Although we were unable to assess the potential dose-response relationship between duration of exposures and increased diarrheal rates, we do not expect that this more detailed information would alter our overall conclusions of an effect of antibiotics on diarrheal rates.

Although we cannot ensure the absence of confounding in this observational study, we have adjusted for detailed treatment indications associated with disease severity that also predict diarrheal risk. As well, because a clinical trial that randomized all antibiotic treatment in this setting would be unethical, we believe this evidence from a well-conducted prospective observational cohort study with good follow-up provides important preliminary evidence toward understanding the impact of antibiotics on diarrheal risk.

Antibiotics are commonly given during infancy at a time in which the developing GI microbiota is most sensitive to perturbations. Although these drugs are lifesaving and should be used for treatment when necessary, the possibility for antibiotics to increase risk of future diarrheal disease should be further explored and potentially considered when making treatment decisions.

\section{ACKNOWLEDGMENTS}

We thank the participants, study staff members, and medical teams for their participation and support. We also thank the study clinic doctors and nurses and Jenipha Elizabeth for help with clinic record data entry.

The parent study was supported by the National Institute of Allergy and Infectious Diseases at the National Institutes of Health (5-R01-AI072222 to H.D.W.). This work was supported by the National Institute of Allergy and Infectious Diseases at the National Institutes of Health (5-T32-AI070114-08 to E.T.R and D43-TW007392 to D.K.). 


\section{REFERENCES}

1. Kutty N. Treating children without antibiotics in primary healthcare. Oman Med J. 2011;26:303-305.

2. World Health Organization. The Rational Use of Drugs in the Management of Acute Diarrhoea in Children. Geneva, Switzerland: World Health Organization; 1990.

3. Diniz-Santos DR, Silva LR, Silva N. Antibiotics for the empirical treatment of acute infectious diarrhea in children. Braz J Infect Dis. 2006;10:217-227.

4. McCaig LF, Besser RE, Hughes JM. Trends in antimicrobial prescribing rates for children and adolescents. JAMA. 2002;287:3096-3102.

5. Vaz LE, Kleinman KP, Raebel MA, et al. Recent trends in outpatient antibiotic use in children. Pediatrics. 2014;133:375-385.

6. Basu S, Chatterjee M, Chandra PK, et al. Antibiotic misuse in children by the primary care physicians-an Indian experience. Niger J Clin Pract. 2008;11:52-57.

7. Kotwani A, Chaudhury RR, Holloway K. Antibiotic-prescribing practices of primary care prescribers for acute diarrhea in New Delhi, India. Value Health. 2012;15(1 Suppl):S116-S119.

8. Mittal SK, Mathew JL. Regulating the use of drugs in diarrhea. J Pediatr Gastroenterol Nutr. 2001;33(Suppl 2):S26-S30.

9. Ministry of Health and Family Welfare G of I. The Drugs and Cosmetics Act 1940 and Rules 1945, 2005. Available at: http://cdsco.nic.in/html/copy\%20 of\%201.\%20d\&cact121.pdf. Accessed October 6, 2013.

10. Directorate General of Health Services. National Policy for Containment of Antimicrobial Resistance India. New Delhi: Ministry of Health \& Family Welfare; 2011.

11. Stewardson AJ, Huttner B, Harbarth S. At least it won't hurt: the personal risks of antibiotic exposure. Curr Opin Pharmacol. 2011;11:446-452.

12. Martin R, Nauta AJ, Ben Amor K, et al. Early life: gut microbiota and immune development in infancy. Benef Microbes. 2010;1:367-382.

13. Hooper LV, Littman DR, Macpherson AJ. Interactions between the microbiota and the immune system. Science. 2012;336:1268-1273.

14. Maynard CL, Elson CO, Hatton RD, et al. Reciprocal interactions of the intestinal microbiota and immune system. Nature. 2012;489:231-241.

15. Sekirov I, Russell SL, Antunes LC, et al. Gut microbiota in health and disease. Physiol Rev. 2010;90:859-904.

16. Buffie CG, Pamer EG. Microbiota-mediated colonization resistance against intestinal pathogens. Nat Rev Immunol. 2013;13:790-801.

17. Jernberg C, Löfmark S, Edlund C, et al. Long-term impacts of antibiotic exposure on the human intestinal microbiota. Microbiology. 2010;156(Pt 11):3216-3223.

18. Dethlefsen L, Relman DA. Incomplete recovery and individualized responses of the human distal gut microbiota to repeated antibiotic perturbation. Proc. Natl. Acad. Sci. U.S.A. 2011;108(Suppl 1):4554-4561.

19. Johnson CL, Versalovic J. The human microbiome and its potential importance to pediatrics. Pediatrics. 2012;129:950-960.

20. Bedford Russell AR, Murch SH. Could peripartum antibiotics have delayed health consequences for the infant? BJOG. 2006;113:758-765.

21. Faa G, Gerosa C, Fanni D, et al. Factors influencing the development of a personal tailored microbiota in the neonate, with particular emphasis on antibiotic therapy. J. Matern Fetal Neonatal Med. 2013;26(Suppl 2):35-43.

22. Saavedra JM, Dattilo AM. Early development of intestinal microbiota: implications for future health. Gastroenterol Clin North Am. 2012;41:717-731.

23. Fujimura KE, Slusher NA, Cabana MD, et al. Role of the gut microbiota in defining human health. Expert Rev Anti Infect Ther. 2010;8:435-454.
24. Wlodarska M, Finlay BB. Host immune response to antibiotic perturbation of the microbiota. Mucosal Immunol. 2010;3:100-103.

25. Guarner F. In: Granger DN, Granger JP, eds. Chapter 5: Dysfunction of the Enteric Microbiota. The Enteric Microbiota. San Rafael, CA: Morgan \& Claypool Life Sciences Publishers; 2012.

26. Chichlowski M, German JB, Lebrilla CB, et al. The influence of milk oligosaccharides on microbiota of infants: opportunities for formulas. Annu Rev Food Sci Technol. 2011;2:331-351.

27. Sela DA, Mills DA. Nursing our microbiota: molecular linkages between bifidobacteria and milk oligosaccharides. Trends Microbiol. 2010;18:298-307.

28. Fernández L, Langa S, Martín V, et al. The human milk microbiota: origin and potential roles in health and disease. Pharmacol Res. 2013;69:1-10.

29. Kattula D, Sarkar R, Sivarathinaswamy P, et al. The first 1000 days of life: prenatal and postnatal risk factors for morbidity and growth in a birth cohort in southern India. BMJ Open. 2014;4:e005404.

30. Division of Child Health and Development. Indicators for Assessing BreastFeeding Practices. Geneva: World Health Organization; 1991.

31. World Health Organization. The treatment of diarrhoea: a manual for physicians and other senior health workers; 2005. Available at: http://www. who.int/maternal_child_adolescent/documents/9241593180/en/index.html. Accessed October 2, 2013.

32. Kuppuswami B. Manual of Socioeconomic Scale (Urban). New Delhi, 32 , Netaji Subhash Marg: Manasayan; 1981.

33. Brick T, Primrose B, Chandrasekhar R, et al. Water contamination in urban south India: household storage practices and their implications for water safety and enteric infections. Int J Hyg Environ Health. 2004;207:473-480.

34. White GC, Bennetts RE. Analysis of frequency count data using the negative binomial distribution. Ecology 1996;77:2549.

35. Greenland S, Pearl J, Robins JM. Causal diagrams for epidemiologic research. Epidemiology. 1999;10:37-48.

36. Ruuska T, Vesikari T. Rotavirus disease in Finnish children: use of numerical scores for clinical severity of diarrhoeal episodes. Scand J Infect Dis. 1990;22:259-267.

37. World Health Organization. WHO Child Growth Standards: Length/height for-Age, Weight-for-Age, Weight-for-Length, Weight-for-Height and Body Mass Index-for-Age, Methods and Development. Geneva, Switzerland: World Health Organization; 2006.

38. Antonopoulos DA, Huse SM, Morrison HG, et al. Reproducible community dynamics of the gastrointestinal microbiota following antibiotic perturbation. Infect Immun. 2009; 77:2367-2375.

39. Young VB, Schmidt TM. Antibiotic-associated diarrhea accompanied by large-scale alterations in the composition of the fecal microbiota. J Clin Microbiol. 2004;42:1203-1206.

40. Looft T, Allen HK. Collateral effects of antibiotics on mammalian gut microbiomes. Gut Microbes. 2012;3:463-467.

41. Maldonado J, Cañabate F, Sempere L, et al. Human milk probiotic Lactobacillus fermentum CECT5716 reduces the incidence of gastrointestinal and upper respiratory tract infections in infants. $J$ Pediatr Gastroenterol Nutr. 2012;54:55-61.

42. Risnes KR, Belanger K, Murk W, et al. Antibiotic exposure by 6 months and asthma and allergy at 6 years: findings in a cohort of 1,401 US children. Am J Epidemiol. 2011;173:310-318.

43. Trasande L, Blustein J, Liu M, et al. Infant antibiotic exposures and earlylife body mass. Int J Obes (Lond). 2013;37:16-23. 